\title{
Neurosis y subjetividad de la época
}

\section{Neurosis and the subjectivity of time}

\section{Miguel Ferrero}

RESUMEN:

En este articulo se intenta dar cuenta de una relación posible entre la ideología producto de un modo de organización socio-económico-politico llamado capitalista, en especial en su versión actual denominada "neoliberalismo", y su relación con un modo de la subjetividad. Relación que se considera necesaria, ya que el "neoliberalismo" se sostiene en el intento de producir y reproducirse en subjetividades caracterizadas por un pensamiento débil, es decir que no pone en juego operaciones lógicas sino que reproduce dichos y opiniones que no cuestionan este modo de producción neoliberal.

Estas subjetividades de la época caracterizadas por lo que Lacán denomina "pasión de la ignorancia", "debilidad mental" en las neurosis y la "locura de creérselas", no son posiciones novedosas en la neurosis; lo novedoso lo podemos encontrar en la manera en que la ideología predominante de la época propicia estas posiciones neuróticas y a su vez estas posiciones se hacen necesarias para sostener esta ideología. En el apartado final "Lo posible y lo contingente" se conjetura acerca de la manera en que esta articulación entre subjetividad y época incide y obstaculiza la práctica analítica.

\section{SUMMARY:}

This paper tries to give an account of a possible relationship between a mode of subjetivity and the ideology product of a socio-political-economical mode of organization called capitalism, especially in its current version called "neoliberalism". A relationship that is considered necessary, since "neoliberalism" is sustained in the attempt to produce and reproduce itself in subjectivities characterized by weak thinking, that is to say that it does not put into play logical operations but reproduces set phrases and opinions that do not question this mode of Neoliberal production.

These current subjectivities, characterized by what Lacan calls: "passion of ignorance", "mental weakness" in neuroses and "madness of belief", are not new positions in the neurosis. The novelty can be found in the way in which this prevailing ideology propitiates these neurotic positions and in turn these positions are necessary to sustain this ideology. In the final section "The possible and the contingent" it is conjectured about the way in which this articulation between subjectivity and ideology impinges and hinders analytical practice. 
Palabras Clave:

Neoliberalismo - Psicoanálisis - Sujeto -

Subjetividad - Ignorancia
WORD PAD:

Neoliberalism - Psychoanalysis - Subject Subjectivity - Ignorance 


\section{INTRODUCCIÓN}

El sutil humor gráfico de Daniel Paz, me permite introducir las razones que encuentro para escribir este artículo. El chiste en cuestión (del suplemento "Radar" del diario "Pagina 12" del 6/I I/2016), se desarrolla en dos escenas, la primera es encabezada por esta observación: “Al señor argentino medio le preocupa que los argentinos tengan trabajo" y debajo de ella hay un señor que le dice a una mujer que vende en la calle: "ustedes los bolivianos vienen a quitarnos el trabajo a los argentinos", a lo que la vendedora responde: "yo soy de Jujuy", y el señor le dice: "no me importa de que país viene". En la segunda escena, encabezada por otro comentario: "Pero a veces le chupa un huevo", allí vemos al señor en su casa diciéndole muy contento a su esposa: "mirá lo que me compré, zapatos italianos y un traje francés", la esposa responde: "igenial!". El alegre señor llamado por Paz: "argentino medio", no encuentra relación entre lo que le dice a la vendedora y lo que le dice a su mujer. Esta incongruencia hace a uno de los núcleos del chiste, diremos que es necesario que en la segunda escena se ignore lo dicho en la primera, ya que es obvio que si lo zapatos y el traje se hacen en Italia y en Francia, esto quita puestos de trabajo a los argentinos. Esta ignorancia es posible en tanto haya un pensamiento que llamaremos: "débil”, el personaje en cuestión no pudo o no quiso poner en relación sus dichos y arribar a partir de ellos a una conclusión que lo interrogase. Si el chiste se produce es porque algún efecto de verdad se pone en juego.

Escucho con cierta frecuencia situaciones como las relatadas, que dejan de ser un chiste, cuando ocurren en el encuentro con un paciente, y nos llevan a largos periodos de entrevistas, que no llegan a ser preliminares a un análisis, en tanto se trata de sujetos que se resisten a encontrarse con sus contradicciones e incongruencias y que por lo contrario reaccionan con disgusto o indiferencia cuando quien los escucha interviene marcándolas. Esto hace que de estos encuentros habitualmente no se produzca un discurso analítico, siendo que dada la estructura neurótica de quien nos consulta podíamos pensar que un análisis era posible.

Estas situaciones han hecho que me interesase en considerar ante qué modos de las neurosis se han consolidado estos sujetos, que no aceptan lo que ponga en juego la diferencia entre enunciado y enunciación, que desisten de la posibilidad de hacer su propia lectura, en consecuencia su interpretación singular. Encuentro un camino posible a seguir, en algunas observaciones que hace Lacan en referencia a lo que llama en el Seminario 1, Los escritos técnicos de Freud "pasión de la ignorancia" (LACAN, I953, p.394), y en el Seminario 24, "L'insu": la "debilidad mental" en la neurosis (LACAN, I977, I I/OI/I977).

Podemos deducir de estas posiciones subjetivas desplegadas en las consultas con alguien llamado psicoanalista, un correlato con lo que ocurre en la vida cotidiana, incluido lo que es dado escuchar y ver en cualquier medio de comunicación masiva, lo cual hace pensar en una relación no casual sino probablemente necesaria, entre lo que se dice y circula a nivel de la comunidad y lo que se oye en el consultorio.

\section{SubjeTIVIDAd de la éPoca}

Recurriré para iniciar este desarrollo a la transitada cita de lo que dice Lacan en "Función y campo de la palabra y del len- 
guaje en el psicoanálisis":

Mejor pues que renuncie quien no pueda unir a su horizonte la subjetividad de su época. Pues ¿como podría hacer de su ser el eje de tantas vidas aquel que no supiera nada de la dialéctica que lo lanza con esas vidas en un movimiento simbólico? (LACAN, I953)

Esta cita presenta la suficiente ambigüedad como para que tengamos que hacer nuestra propia lectura y no se transforme en un slogan lacaniano mas.

En principio: ¿qué podríamos entender por "época?, sabemos como definición general, que época hace referencia a un periodo de tiempo marcado por un comienzo, dado por algún acontecimiento determinado y al que sigue un tiempo cuyo transcurrir implica un fin, de época; ahora bien: ¿cuándo comenzó nuestra época?, ¿ese periodo implica un "para todos"?, ¿qué caracteriza a esta época?, por lo tanto ¿qué la diferencia de otras?, ante semejante extensión de criterios posibles, haré una elección acotada y me referiré a época en tanto la actualidad de mi circunstancia, compartida con otros que habitan el mismo mapa simbólico, lo cual incluye un modo de producción económico y de vínculos sociales llamado capitalista. Ahora bien si consideramos, tal como nos propone Lacan que una época determina subjetividades, una primera aproximación a esta idea podría ser pensar "subjetividad de la época", como la manera en que es conceptualizado el sujeto en determinado momento histórico, entonces podríamos concluir provisoriamente que esa subjetividad, está caracterizada por los ideales del yo y en consecuencia las maneras que adquieren los imperativos superyoicos, lo que es correlativo a cómo es tratado el malestar, cómo es tratado lo diferente y la diferencia, lo particular y lo general, en fin qué lugar se le hace a la castración y a la división del sujeto tal como lo considera el psicoanálisis. Aunque ¿habrá habido época en que este sujeto dividido haya tenido lugar?, evidentemente el siglo $\mathrm{xx}$ vio nacer el psicoanálisis, pero ¿eso implica necesariamente un lugar a nuestro sujeto en la cultura de la época?

Con lo expuesto anteriormente, trato de establecer una diferencia decisiva entre sujeto y subjetividad. La subjetividad la ubico en el registro del yo y sus vasallajes, por lo cual se puede deducir que la subjetividad social construye personas e impone modelos, lo que hace obstáculo justamente a las operaciones subjetivas, pero sin olvidar que la existencia de lo subjetivo impide que haya una subjetividad de época consolidada, ya que eso es a lo que llamamos "el malestar en la cultura", ese malestar que no cesa de insistir y hace que mientras haya sujeto, la producción de subjetividades vaya a encontrar sus fisuras.

En lo que hace a nuestra actualidad, el aspecto que creo decisivo en la construcción de subjetividad, es la característica que ha tomado el modo de producción capitalista, al que se ha dado en llamar "neoliberalismo", al cual en términos absolutamente generales podríamos definir como lo que caracteriza a un aparato de estado que no regula al mundo financiero y a la empresas multinacionales sino que es regulado por ellas. Susana Murillo en Colonizar el dolor lo dice de esta manera: "el neoliberalismo es una estrategia del sistema capitalista, cuyo núcleo es proponer un individuo solitario, centrado en si mismo y estimulado a la competencia, que 
busca resolver la angustia de su propia finitud a través del consumo"(Murillo, 2008).

De esta cita podemos deducir que el proyecto neoliberal sería construir subjetividades que no solo no obstaculicen los objetivos económicos sino que sean además funcionales a esos objetivos. De estas conjeturas se deduce que si la época construye subjetividades, su correlato es la construcción de una "realidad" consecuente con ella. Me refiero a "realidad" en tanto una visión del mundo compartida, en tanto este aspecto de la realidad sea una visión portadora de valores e ideales que definen como debe y/o debería funcionar lo social, lo económico, lo político, por lo tanto es portadora de un saber y una verdad, a este carácter de la realidad es a lo que llamamos: ideología. Es decir, a la suma de significados y sentidos que consolidan nuestro ser en el mundo, hacen lazo con los semejantes que comparten esta visión, crean consensos e inevitablemente segregan a quienes ponen en dudas esa manera de organizar la realidad, ante la posibilidad de que lo diferente produzca desorden en el orden simbólico e imaginario creado. La adhesión a una ideología, la naturalización de un determinada visión del mundo, se hacen más consistentes en la medida en que la trama discursiva sea mas frágil o por las situaciones sociales del momento o por la fragilidad en la constitución del yo de un sujeto determinado. Nos encontramos así que de estas fragilidades surgen creencias transformadas en fanatismos y odios que viran a la destrucción de lo distinto que desestabilice la unidad de esa visión del mundo, dicho de otra manera, que ponga en cuestión el "status quo".

Realidad, ideología, son términos que intento separar pero con el objetivo de una mayor claridad, ya que la escena de la realidad en la que se mueve un sujeto, no podría ser ajena a los argumentos con que se explica esa realidad. Por ejemplo: la explicación de la realidad que sostiene el neoliberalismo, crea la idea de que un sujeto encontraría su ser y su felicidad en ser "empresario de si mismo", el "self made man" ("el hecho a si mismo"), cuya salud, educación, en fin su calidad de vida, no son cuestiones de estado sino de logros personales. (A veces en psicoanálisis confundimos "responsabilidad del sujeto", con esta ideología de la autonomía personal que en mayor o menor medida nos atraviesa). Habría que agregar al menos un aspecto de esta ideología imperante, el tratamiento que se hace del goce, "hágalo ya”, "téngalo ya”, “igoce ya!”, con que se intenta entretenernos, lo que se acompaña de "no deje de consumir", claro, que el mismo objeto para todos, (al menos en los países en que esto es posible), tratamiento que intenta borrar la falta, la contingencia de los objetos de goce, la insatisfacción constitutiva de lo humano, en consecuencia borrar lo que nos hace humanos: la singularidad del deseo. Por otra parte si lo valorado es el éxito en relación al logro individual de bienes y goces, el fracaso no tiene lugar, es lo que se debería evitar, desde nuestra lectura: evitar la castración.

Si bien toda constitución de la realidad es el modo de armar una ficción que nos aparta de lo real y nos permite la vida, dicho en términos de Lacan, si bien "no hay discurso que no sea de la apariencia", por lo tanto no hay discurso de lo real, en lo que hace a nuestro presente encuentro en el discurso de derecha actual, una concepción del sujeto que necesita de determinadas operaciones denegatorias y renegato- 
rias que concluyen en el "no querer saber" que el saber no es de lo real.

\section{No QUERER SABER}

El "no querer saber" encuentra su sostén privilegiado en los medios de comunicación y redes sociales, cuya proliferación y globalización, resultan inéditos. Basta encender el televisor para encontrarnos con el denodado intento de que no se piense; puede un entrevistado estar diciendo un enunciado y al unísono ser desmentido por el zócalo; se contará con que, o se escucha o se lee de manera que lo uno excluye lo otro, ya que lo oído y lo leído, muchas veces no tiene ninguna coincidencia. Otro ejemplo paradigmático del desconocimiento de la capacidad de juicio del televidente, lo encontramos en la confusión que se crea entre mostrar y demostrar, por ejemplo: en un programa periodístico se muestra una maqueta de un bunker donde supuestamente se guarda dinero, dándole existencia real a tal bunker en tanto un periodista lo da como verdad revelada...jpor una maqueta!, la supuesta semejanza visual y la autoridad del periodista bastan como criterios de verdad, podríamos agregar otro efecto característico: la confusión o mejor la fusión entre la persona y el argumento, un dicho es validado o no según el prejuicio que se tenga sobre quien lo dice. Me valdré nuevamente de la agudeza de Daniel Paz: en uno de sus chistes ("Pagina 12 " de I8/3/20I7), vemos a un hombre que abre la puerta, esta mojado, y le dice a su mujer: “¡No sabes como esta lloviendo afuera!”, a lo que ella, viendo la televisión le responde: "pero si la tele dice que está soleado", el hombre azorado contesta: "Uy, me mojé al pedo"; frente a la tele no hay hechos ni razones, es incuestionable, lo mojado... es seco.
Destacamos en este chiste otra operación decisiva, implícita en lo que dice la mujer: "si la tele dice...", esta operación es la desestimación: "no te creo cuando me desengañas, por mas mojado que yo te vea, elijo el engaño de la televisión: ¡no llueve!"

Encuentro, como fue dicho al comienzo, en las caracterizaciones que hace Lacan de la debilidad, de la ignorancia como pasión, y en la locura de creérselas, modos de la neurosis, que me dejan el interrogante de si es que acaso no responden más a rasgos de carácter, es decir a fijaciones pulsionales a ideales en las que el yo se sostiene, que a lo que podríamos pensar como síntomas o inhibiciones. Lacan reitera en relación a estas formaciones del yo una cuestión que destaco: "no querer saber otra cosa que la que ya se sabe", aunque sea esta la necesidad de todo neurótico, cuyo guión fantasmático se sostiene en no querer saber de la castración, esto está siempre en tensión con el hecho de querer saber qué quiere el Otro; pero en el "no querer saber" al que me refiero, podemos considerar un falla en la función de la creencia, en tanto la contingencia que hace a la relación entre verdad y saber, desestabiliza al sujeto, quien encuentra su anclaje en la resistencia a la duda, pues si el saber no es la verdad entonces: “¿quien soy?”. El "soy" en el lugar del "pienso" se ha organizado de manera tan consistente, que implica una consolidación del carácter, que no en vano está incluida entre las pasiones del ser, las locuras neuróticas, y las debilidades que impide un análisis.

En tanto terquedad y sordera resultan inconmovibles, estas fijaciones en las neurosis contribuyen a constituir un campo propicio, para consolidar la ideo- 
logía neoliberal. Aunque es indudable que cualquier ejercicio del poder de uno o unos individuos o instituciones, para sostenerse necesita del rechazo al cuestionamiento, la"subjetividad de nuestra época" se caracteriza especialmente por el mandato de "no piense, consuma y goce", "no piense, que otro lo hará por Ud", y aquí nos encontramos con la otra posición subjetiva necesaria, la del canalla, el que usa a quien no quiere saber para lograr sus fines utilitarios, en términos cotidianos podemos decir que: "el necio hace una buena pareja con el sinvergüenza".

En relación a lo que hace a nuestra práctica, podemos caracterizar a quienes sostienen el rechazo a interrogar el saber que poseen y que los posee, sujetos que ven amenazada su identidad, su unidad, por lo que interroga su ser y el ser de lo, y los, que lo rodean.

La diferencia entre el yo y lo otro es débil, lo que lleva a una continuidad que debe ser mantenida ante el peligro de caer en un desconcierto angustiante, esto lleva a que el odio sea el afecto que produce el o lo que interroga al saber petrificado, es así como la xenofobia y la segregación del diferente son en general los matices que adquiere el odio. Si la neurosis es la estructura privilegiada para que los efectos de sugestión se produzcan, el apasionado por ignorar tiene una disposición especial para deponer el tiempo de comprender y el momento de concluir, en quienes ocupan el lugar de líderes que aseguren que nada va a cambiar y "las cosas son así desde que el mundo es mundo" y que "todo esta dicho, y no hay mas nada que agregar", frases tan dichas y oídas que ilustran momentos o unos estados permanentes de las neurosis. Si de lo que se trata en una cura analítica es de mantener la mayor distancia entre el ser y el Uno, esto es correlativo a mantener la mayor distancia entre el ser de un significado y el uno de un sentido único, lo que abre a otros sentidos hace que se pierda el referente, con lo cual lo que es dicho puede ser pensado de otra manera, no querer saber de la pluralidad de sentidos, es oponerse a esta operación que es el análisis mismo.

\section{LO POSIBLE Y LO CONTINGENTE}

Cuando esto ocurre y persiste, es difícil recordar a un sujeto el decir que hay en sus dichos, la sensibilidad al significante y a las operaciones simbólicas quedan depuestas, los efectos de la función de la falta no encuentran lugar, con lo cual el brillo fálico se apaga. De ello se deduce el tedio, la abulia y la falta de entusiasmo con que nos encontramos en las consultas de quienes: o creen "estar de vuelta" o se desconciertan ante la intervención mínima dada por una pregunta.

Lacan sostiene más de una vez que es inútil intentar un análisis con sujetos que Freud no hubiese dudado en rechazar. No obstante el pesimismo de Lacan, considero que si un sujeto consulta por un sufrimiento aunque este se sostenga solo en quejas y a pesar de no escucharse conflictos manifiestos, y no obstante este sujeto esté dispuesto a ser escuchado, aunque no interrogado, y si la disposición de quien escucha se lo permite, quizá haya que dar tiempo a que en algún momento, hipotéticamente, pueda escuchar que es escuchado y escucharse; lo cual si bien no tiene por qué constituirse en un análisis, permite considerar que es posible que una intervención pueda abrir una posibilidad insospechada para los 
dos sujetos que se encuentran en esa entrevista.

Si es que hay transferencia de amor aunque no se haya constituido el sujeto supuesto saber, que un sujeto que sufre se sienta alojado y respetado en los recursos que encontró para vivir, creo que no es poco ofrecer una escucha aunque en principio, no pase de eso, la disposición de animo de quien preste su oreja decidirá hasta cuando esperar. Porque una manera de no querer saber, es tratar de ignorar, lo que no podríamos ignorar: que en relación al futuro solo podemos conjeturar, de hecho no sabemos de lo que vendrá.( Los dichos de Lacan obviamente no son adivinatorios, en fin, la teoría no dirige la cura, tampoco prescribe modos de vida, como a veces, para nuestra tranquilidad, quisiéramos creer).

Pero para ello se necesita de parte de quien se diga psicoanalista, que deje de lado "la gesta psicoanalítica", uno de los modos que tiene la pasión por la ignorancia en nuestra practica, el hecho de no querer saber que la práctica psicoanalítica tiene sus límites.

Para concluir y en relación a lo anteriormente dicho, agregaré que otro modo de la pasión por la ignorancia que se presenta en nuestra practica, y de la no que deberíamos dejar de estar advertidos, es reflexionar acerca de cómo nos atraviesa la moral neoliberal: por ejemplo cuando confundimos responsabilidad del sujeto con "hacerse cargo" a nivel conciente, o cuando consideramos al psicoanálisis como un bien de consumo, o cuando confundimos los logros de un análisis con los ideales del "hecho a si mismo" y por si mismo, independiente de toda facilitación social, es decir el supuesto valerse por sí mismo a despecho de las condiciones socio-políticas, o peor confundimos los logros de un análisis con la consecución de bienes de consumo. En relación a esto y para concluir, recuerdo la frase que nos dejó Lacan para pensar, dijo, en el Seminario "La Ética" dirigiéndose a los analistas: "No hay ninguna razón para que nos hagamos garantes de sostener el ensueño burgués" (LACAN, I969, p.362). Podemos agregar: ni ningún otro ensueño que impida despertar.

\section{REFERENCIAS BIBLIOGRÁFICAS}

LACAN, J. (I953-54). "La verdad surge de la equivocación”, Seminario 1: Los escritos técnicos de Freud, Buenos Aires, Paidós.

$$
\text { (1959-1960) Seminario 7: La }
$$

Ética del psicoanálisis. Buenos Aires, Paidós.

(I97I) Seminario 18: De

undis-curso que no sería de la apariencia. Buenos Aires, Paidós.

$$
\text { (1976-1977) Seminario 24: }
$$

L'insu. Versión inédita. (I946) "Acerca de la causalidad psíquica" en Escritos I. Buenos Aires, Siglo XXI Editories.

Murillo, S: (2008) Colonizar el dolor. Buenos Aires, Clasco.

PAz, D (06/I I/20I6). "El Nudo Infinito". Página 12. Recuperado de "Cogito e historia de la locura", en La escritura y la diferencia. Barcelona, Antrhopos. 


\section{RESEÑA CURRICULAR DEL AUTOR}

Miguel Ferrero practica el psicoanálisis desde I978. Fue miembro de la Escuela de Psicoanálisis Sigmund Freud de Rosario durante 30 años, nominado AME. Participó y participa como supervisor, coordinador y dictante de seminarios en distintas instituciones públicas. Escribió artículos para diferentes publicaciones psicoanalíticas. Es dictante del Seminario: "Posibilidad y obstaculos en la cura psicoanalítica”, de la Maestría en Psicoanálisis, Facultad de Psicología, UNR. 